ISAHP 1996, Vancouver, Canada, July 12-15, 1996

\title{
ON THE SOLUTION OF A SUPERHIERARCHY WITH A BAYES LOOP
}

\author{
Bochang Chen ${ }^{\ddagger}$ and V.M. Rao Tummala ${ }^{\S}$ \\ Department of Manufacturing Engineering \\ City University of Hong Kong, Kowloon, Hong Kong
}

\begin{abstract}
In this paper, we discuss the uniqueness condition for the solution of a kind of a superhierarchy and establish the necessary and sufficient conditions for a superhierarchy to be a Bayes loop. Also, a simple method of finding the solution is introduced.
\end{abstract}

\section{Introduction}

The problem of a superhierarchy with a Bayes loop was introduced in the literature by Saaty (Saaty, 1994). This problem considers a network as shown in Figure 1 , where $L_{1}=\left\{\theta_{1}, \theta_{2}, \ldots, \theta_{n}\right\}$ is the state space and $L_{2}=\left\{x_{1}, x_{2}, \ldots, x_{m}\right\}$ is the sample space. Let $P_{1}=\left(\left(p_{1}\right)_{1},\left(p_{1}\right)_{2}, \ldots,\left(p_{1}\right)_{n}\right)^{T}$ be the importance vector of $L_{1}$ given $G_{1}$, and $P_{2}=\left(\left(p_{2}\right)_{1},\left(p_{2}\right)_{2}, \ldots,\left(p_{2}\right)_{m}\right)^{T}$ be the importance vector of $L_{2}$ given $G_{2}$. The elements of $P_{1}$ and $P_{2}$ are all positive (the elements with zero components'can be deleted). The interimpact between $L_{1}$ and $L_{2}$ is described by two matrices $P_{12}$ and $P_{21}$, respectively, where $P_{12}=\left(\left(p_{12}\right)_{i j}\right)$ is the column stochastic matrix whose jth column $\left(\left(p_{12}\right)_{i j},\left(p_{12}\right)_{2 j}, \ldots,\left(p_{12}\right)_{\mathrm{nj}}\right)^{\mathrm{T}}$ is the importance vector of $L_{1}$ given $x_{j}$ of $L_{2}$, and $P_{21}=\left(\left(p_{21}\right)_{i j}\right)$ is the column stochastic matrix whose $j$ th column $\left(\left(p_{21}\right)_{1 j},\left(p_{21}\right)_{2 j}, \ldots\right.$, $\left.\left(\mathrm{p}_{21}\right)_{\mathrm{mj}}\right)^{\mathrm{T}}$ is the importance of $L_{2}$ given $\theta_{j}$ of $L_{1}$. Naturally, $P_{12}, P_{21}$ are both non-negative matrices.

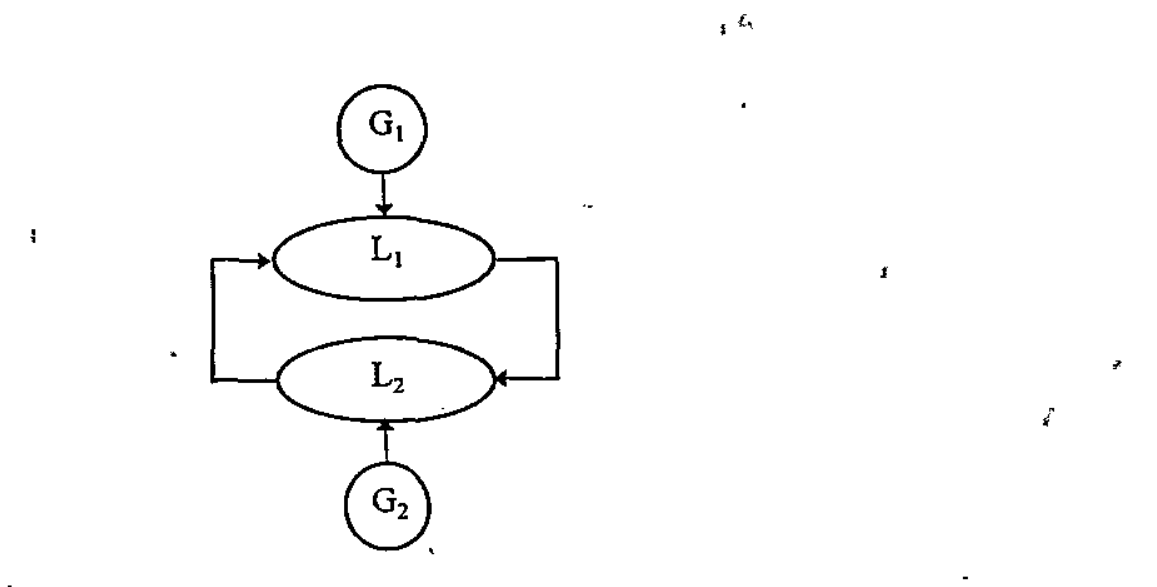

Figure 1: A Superhierarchy with a Bayes Loop

The supermatrix corresponding to the superhierarchy of Figure 1 is given by

\footnotetext{
$\ddagger$ On leave from School of Management, Fudan University, Shanghai, China

${ }^{\S}$ On leave from College of Business, Eastern Michigan University, Ypsilanti, MI, USA
} 


$$
\begin{aligned}
& \begin{array}{lllll}
L_{1} & L_{2} & G_{1} & G_{2}
\end{array} \\
& \mathrm{~W}=\begin{array}{l}
\mathrm{L}_{1} \\
\mathrm{~L}_{2} \\
\mathrm{G}_{1} \\
\mathrm{G}_{2}
\end{array}\left(\begin{array}{cccc}
0 & \mathrm{P}_{12} & \mathrm{P}_{1} & 0 \\
\mathrm{P}_{21} & 0 & 0 & \mathrm{P}_{2} \\
0 & 0 & 0 & 0 \\
0 & 0 & 0 & 0
\end{array}\right)
\end{aligned}
$$

This kind of superhierarchy is used for feedback decision problems appeared in many areas. A decision making problem that doctors often face, for example, is how to diagnose the disease of a patient in determining a medical treatment plan according to the patient's symptoms. In this problem, the diseases cause some symptoms, and the symptoms reflect some diseases; that is, there exists a feedback between diseases and symptoms. The structure of these kinds of decision problems can be shown as in Figure 2.

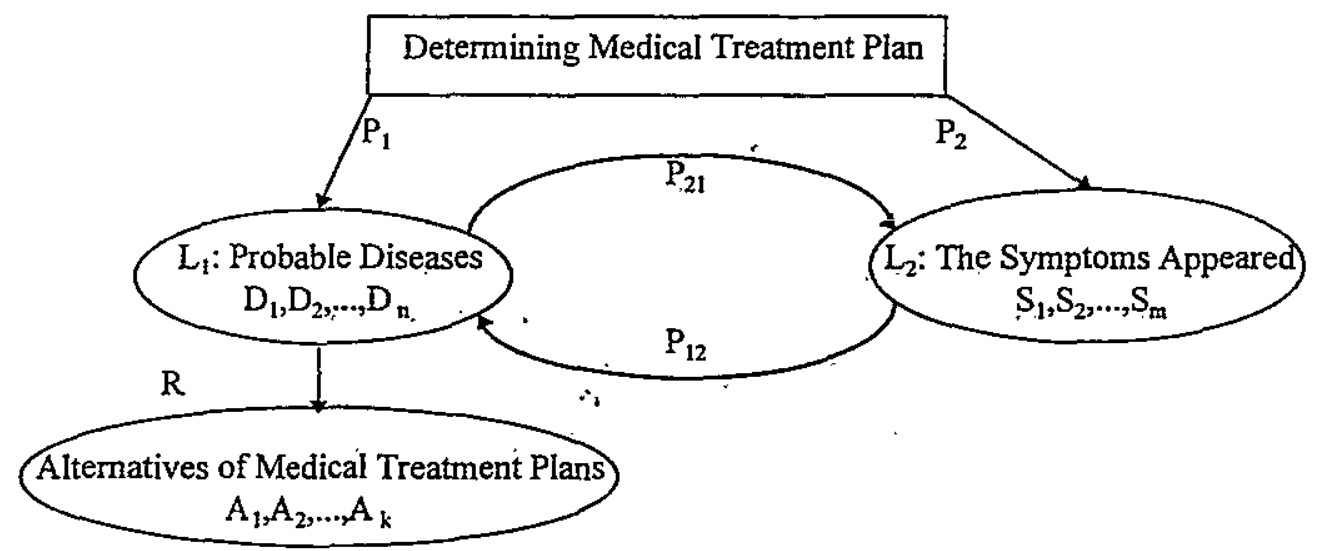

Figure 2: The Superhierarchy with a Bayes Loop for Medical Decision Problems

Here, $p_{1}=\left(\left(p_{1}\right)_{1},\left(p_{1}\right)_{2}, \ldots,\left(p_{1}\right)_{n}\right)^{T}$ is the priority weights. vector for the diseases of the patient for whom a medical treatment plan will be selected, and $P_{2}=\left(\left(p_{2}\right)_{1},\left(p_{2}\right)_{2}, \ldots,\left(p_{2}\right)_{m}\right)^{T}$ is the priority weights vector for the symptoms of the patient. The inter-impact between $L_{1}$ and $L_{2}$ is described by two matrices $P_{12}$ and $P_{21}$, where the jth column of $P_{12}=\left(\left(p_{12}\right)_{i j}\right)_{n \times m}$ is the priority weights vector of the diseases for $a$ given symptom $S_{j}, j=1,2, \ldots, m$. Similarly, the jth column of the matrix $P_{21}=\left(\left(p_{21}\right)_{i j}\right)_{m \times n}$ is the priority weights vector of the symptoms for a given decease $D_{j}, j=1,2, \ldots, n$. And the jth column of the matrix $\mathrm{R}=\left(\mathrm{r}_{\mathrm{ij}}\right)_{\mathrm{kxm}}$ is the priority weights vector of the alternatives of medical treatment plans for a given disease $D_{j}, j=1,2, \ldots, n$.

Obviously, $P_{1}$ and $P_{2}$ are the solutions of the following equation

$$
\mathrm{P}_{12} \mathrm{~b}=\mathrm{a} \text { and } \mathrm{P}_{21} \mathrm{a}=\mathrm{b}
$$

for some positive vectors $a=\left(a_{1}, a_{2}, \ldots, a_{n}\right)^{\top}$ and $b=\left(b_{1}, b_{2}, \ldots, b_{m}\right)^{T}$. The decision problem in medical diagnosing cases is to find $P_{1}$ and $P_{2}$ from Eq. (1) given $P_{12}$ and $P_{21}$ which are obtained by the doctor according to his or her medical knowledge, and then to determine the corresponding medical treatment plan. The solution of Eq. (I), however, is not always unique. Consider the following example.

Example 1: Define 


$$
\mathrm{P}_{21}=\left(\begin{array}{cccc}
.8 & .3 & 0 & 0 \\
.2 & .7 & 0 & 0 \\
0 & 0 & .6 & .3 \\
0 & 0 & .4 & .7
\end{array}\right) \text { and } \mathrm{P}_{12}=\left(\begin{array}{cccc}
.6400 & .1600 & 0 & 0 \\
.3600 & .8400 & 0 & 0 \\
0 & 0 & .9474 & .8372 \\
0 & 0 & .0526 & .1628
\end{array}\right)
$$

We can find vectors $\bar{a}, \bar{b}, \widetilde{a}$ and $\widetilde{b}$ as

$$
\overline{\mathrm{a}}=\left(\begin{array}{l}
.080 \\
.120 \\
.720 \\
.080
\end{array}\right), \overline{\mathrm{b}}=\left(\begin{array}{l}
.100 \\
.100 \\
.456 \\
.344
\end{array}\right) \text { and } \widetilde{\mathrm{a}}=\left(\begin{array}{l}
160 \\
.240 \\
.540 \\
.060
\end{array}\right), \widetilde{\mathrm{b}}=\left(\begin{array}{l}
.200 \\
.200 \\
.342 \\
.258
\end{array}\right)
$$

such that both satisfy Eq. (1). In fact, for any $\lambda \in[0,1]$

$$
\mathrm{a}=\lambda\left(\begin{array}{l}
.4 \\
.6 \\
0 \\
0
\end{array}\right) \div(1-\lambda)\left(\begin{array}{l}
0 \\
0 \\
.9 \\
.1
\end{array}\right), \mathrm{b}=\lambda\left(\begin{array}{l}
.5 \\
.5 \\
0 \\
0
\end{array}\right)+(1-\lambda)\left(\begin{array}{c}
0 \\
0 \\
.57 \\
.43
\end{array}\right) \text {. }
$$

constitutes a solution of Eq. (1).

Besides, in many cases, the solution of Eq. (1) also satisfy

$$
\left(p_{12}\right)_{i j} b_{j}=\left(p_{21}\right)_{j i} a_{i} \quad i=1,2, \ldots, n ; j=1,2, \ldots, m .
$$

when we call the inter-impact between $L_{1}$ and $L_{2}^{i}$ is a Bayes loop. In the diagnostic problem of Figure 2 , for example, $\left(\mathrm{p}_{12}\right)_{\mathrm{ij}}\left(\mathrm{p}_{2}\right)_{j}$ and $\left(\mathrm{p}_{21} \mathrm{i}_{\mathrm{j} j}\right)\left(\mathrm{p}_{\mathrm{i}}\right)$ are the priority weights of the patient suffering from disease $\mathrm{D}_{\mathrm{i}}$ and appearing with symptom $S_{j}$, respectively, such that .

$$
\left(p_{12}\right)_{i j}^{\prime}\left(p_{2}\right)=\left(p_{21}\right)_{j i}\left(p_{1}\right)_{i} \quad i=1,2, \ldots, n ; j=1,2, \ldots, m .
$$

In this paper, we shall first discuss the uniqueness condition of the solution of Eq. (1), then establish the necessary and sufficient conditions for the superhierarchy in Figure 1 to be a Bayes loop. We shall also describe a simple method of finding the unique vectors $a$ and $b$ that satisfy Eq. (1) of Bayes loop.

\section{The Uniqueness Condition of the Solution of Eq. (1)}

We begin with some definitions.

Definition 1: The inter-impact between $L_{1}$ and $L_{2}$ is called a Bayes loop if

$$
\left(p_{12}\right)_{i j}\left(p_{2}\right)_{j}=\left(p_{21}\right)_{j i}\left(p_{\nu}\right)_{i} \quad \text { for } i=1,2, \ldots, n ; j=1,2, \ldots, m
$$

Definition 2: If for any fixed $i \in\{1,2, \ldots, n\}$ and $j \in\{1,2, \ldots, m\},\left(p_{12}\right)_{i j}>0$ implies $\left(p_{21}\right)_{j i}>0$ and vice versa, we call the inter-impact between $L_{l}$ and $L_{2}$ has Property $A$. 
Because $\left(p_{1}\right)_{i}(i=1,2, \ldots, n)$ and $\left(p_{2}\right)_{j}(j=1,2, \ldots, m)$ are all positive, from Definition 1 we see that Property $A$ will be satisfied when the inter-impact between $L_{1}$ and $L_{2}$ is a Bayes loop.

Definition 3: The inter-impact between $L_{1}$ and $L_{2}$ is called reducible if $L_{1}$ and $L_{2}$ can be split into two non-empty complementary subsets $L_{l a} L_{l b}$ and $L_{2 a} L_{2 b}$, respectively, such that

$$
\left(p_{12}\right)_{i j}=\left(p_{22}\right)_{j i}=0 \quad \text { for } i \in L_{j a} \text { and } j \in L_{2 b} \text {, or } i \in L_{l b} \text { and } j \in L_{2 a}
$$

Otherwise, the inter-impact between $L_{1}$ and $L_{2}$ is called irreducible. We now state and prove the theorem about the uniqueness of the solution of Eq. (1).

Theorem 1: If the inter-impact between $L_{1}$ and $L_{2}$ is irreducible with Property $A$, then there exist two unique positive vectors $a$ and $b$ that satisfy $E q$. (1); that is,

$$
P_{12} b=a \text { and } P_{2 J} a=b
$$

Proof: Eq. (1) can be rewritten as

Let

$$
\left(\begin{array}{cc}
0 & P_{12} \\
P_{21} & 0
\end{array}\right)\left(\begin{array}{l}
a \\
b
\end{array}\right)=\left(\begin{array}{l}
a \\
b
\end{array}\right)
$$

$$
U=\left(\begin{array}{cc}
0 & P_{12} \\
P_{21} & 0
\end{array}\right)
$$

The inter-impact between $L_{1}$ and $L_{2}$ is irreducible implies the matrix $U$ is irreducible. In fact, if $U$ is reducible, then $U$ can be partitioned (by permutation) into the form

$$
\left(\begin{array}{cc}
A_{1} & 0 \\
B & A_{2}
\end{array}\right)
$$

where $A_{1}$ and $A_{2}$ are square matrices. However, $B$ in Eq.(3) must be zero because of Property A. Thïs means the inter-impact between $L_{1}$ and $L_{2}$ is reducible, which is contrary with the condition of this theorem. So $\mathrm{U}$ must be irreducible. Then, we prove that

$$
\mathrm{U}^{2}=\left(\begin{array}{cc}
\mathrm{P}_{12} \mathrm{P}_{21} & 0 \\
0 & \mathrm{P}_{21} \mathrm{P}_{12}
\end{array}\right)
$$

is regular, or its diagonal mafrices $P_{12} P_{21}$ and $P_{21} P_{12}$ are both primitive. Let $T=\left(t_{i j}\right)=P_{12} P_{21}$. First, the diagonal elements of $T$ must be all positive. In fact, there exists at least one positive element in the ith column of $P_{21}$. Let us denote this element as $\left(p_{21}\right)_{\text {hi }}$. Using Property $A$, we have

$$
\therefore t_{\mathrm{ii}}=\sum_{\mathrm{k}=1}^{\mathrm{m}}\left(\mathrm{p}_{12}\right)_{\mathrm{ik}}\left(\mathrm{p}_{21}\right)_{\mathrm{ki}} \geq\left(\mathrm{p}_{12}\right)_{\mathrm{ih}}\left(\mathrm{p}_{21}\right)_{\mathrm{hi}}>0
$$

Secondly, since the irreducibility of the inter-impact between $L_{1}$ and $L_{2}$, for any fixed $i, j \in\{1,2, \ldots, n\}$ there exists an integer $q$ such that $t_{i j}^{(q)}>0$, where $t_{i j}^{(q)}$ is the element of $T^{q}$. Consequently

$$
t_{i j}^{(q+i)}=\sum_{k=1}^{n} t_{i k}^{(q)} t_{k j} \geq t_{i j}^{(q)} t_{j j}>0
$$

Therefore, $t_{i j}^{(u)}>0$ for any integer $u \geq q$. It means that there exists an integer $r$ such that $T^{s}>0$, which yields $T$ as primitive (Gantmacher, 1977, Vol.II, p.80, Theorem 8). Similarly, $P_{21} P_{12}$ is primitive. The primitivity of $\mathrm{P}_{12} \mathrm{P}_{21}$ and $\mathrm{P}_{21} \mathrm{P}_{12}$ means that $\mathrm{U}^{2}$ is regular or the cyclicity of $U$ is 2 . Thus, any column of the matrix

$$
\tilde{U}=\lim _{\mathrm{N} \rightarrow \infty} \frac{1}{N} \sum_{\mathrm{k}=1}^{\mathrm{N}} \mathrm{U}^{\mathrm{k}}=\frac{1}{2}(\mathrm{E}+\mathrm{U})\left(\mathrm{U}^{2}\right)^{\infty}=\frac{1}{2}\left(\begin{array}{cc}
\left(\mathrm{P}_{12} \mathrm{P}_{21}\right)^{\infty} & \mathrm{P}_{12}\left(\mathrm{P}_{21} \mathrm{P}_{12}\right)^{\infty} \\
\mathrm{P}_{21}\left(\mathrm{P}_{12} \mathrm{P}_{21}\right)^{\infty} & \left(\mathrm{P}_{21} \mathrm{P}_{12}\right)^{\infty}
\end{array}\right)
$$


is identical to any other column (Gantmacher, 1977, Vol.II, p.98). Let a be any column of $\left(\mathrm{P}_{12} \mathrm{P}_{21}\right)^{-}$and $b$ be any column of $\left(\mathrm{P}_{21} \mathrm{P}_{12}\right)^{-\infty}$, respectively. They are both positive and uniquely satisfy Eq. (2) or Eq. (1) (Gantmacher, 1977, Vol.II, p.93 and p.98). This completes the proof.

Since Property A is true for a Bayes loop, we can also obtain the following corollary from Theorem 1.

Corollary 1: If the inter-impact between $L_{1}$ and $L_{2}$ is an irreducible Bayes loop, then there exist two unique positive vectors $a$ and $b$ that satisfy $E q$. (1).

\section{The Equivalent Conditions Between Eq. (1) and Definition 1}

It is easy to see that the solution of Definition 1 is a solution of Eq. (1), but the solution of Eq. (1) is not always a solution of Definition 1 for arbitrary $P_{12}$ and $P_{21}$ with the conditions of Theorem 1 . When would the solution of Eq. (1) be a solution of Definition 1? Or, given $P_{21}$ and $P_{12}$, how can we find that the inter-impact between $L_{1}$ and $L_{2}$ is a Bayes loop? Theorem 2 answers this question. Also, a simple method of finding the solution of Eq. (1) will be introduced. First we introduce two lemmas for Theorem 2.

Lemma 1: When the inter-impact between $L_{1}$ and $L_{2}$ is irreducible with Property $A$, the solution of $E q$. (I) is a solution of Definition 1 if and only if, for coefficient matrices $P_{12}$ and $P_{21}$, there exist positive constants $\alpha_{1}, \alpha_{2}, \ldots, \alpha_{n}$ and $\beta_{1}, \beta_{2}, \ldots, \beta_{m}$ such that.

and

$$
\alpha_{k}\left(p_{12}\right)_{i j}\left(p_{2 I}\right)_{j k}=\alpha_{i}\left(p_{12}\right)_{k j}\left(p_{2 I}\right)_{j i} \quad \text { for } i, k=1,2, \ldots, n ; j=1,2, \ldots, m
$$

$$
\beta_{k}\left(p_{21}\right)_{i j}\left(p_{12}\right)_{j k}=\beta_{i}\left(p_{21}\right)_{k j}\left(p_{12}\right)_{j i} \quad \text { for } i, k=1,2, \ldots m ; j=1,2, \ldots, n
$$

Proof (Necessity): If the solution of Eq. ( 1 ) is a solution of Definition 1 , then the positive vectors $a=$ $\left(a_{1}, a_{2}, \ldots, a_{n}\right)^{T}$ and $b=\left(b_{1}, b_{2}, \ldots, b_{m}\right)^{T}$ satisfy

and

$$
\left(p_{12}\right)_{i j} b_{j}=\left(p_{21}\right)_{j i} a_{i} \quad \text { for } i=1,2, \ldots, n ; j=1,2, \ldots, m
$$

$$
\left(p_{21}\right)_{j k} a_{k}=\left(p_{12}\right)_{k j} b_{j} \quad \text { for } k=1,2, \ldots, n ; j=1,2, \ldots, m
$$

Multiplying both sides of Eq.(7) by the corresponding sides of Eq.(8), we have

$$
a_{k}\left(p_{12}\right)_{i j}\left(p_{21}\right)_{j k}=a_{i}\left(p_{12}\right)_{k j}\left(p_{21}\right)_{j i} \quad \text { for } i, k=1,2, \ldots, n ; j=1,2, \ldots, m
$$

For the same reason, we also have

$$
b_{k}\left(p_{21}\right)_{i j}\left(p_{12}\right)_{j k}=b_{i}\left(p_{21}\right)_{k j}\left(p_{12}\right)_{j i} \quad \text { for } i, k=1,2, \ldots, m ; j=1,2, \ldots, n
$$

Thus, the necessity follows.

(Sufficiency): Assume that there exist positive constants $\alpha_{1}, \alpha_{2}, \ldots, \alpha_{n}$ and $\beta_{1}, \beta_{2}, \ldots, \beta_{m}$ such that Eqs. (5) and (6) are true. Summing up Eq.(5) with respect to k, we obtain

$$
\left(p_{12}\right)_{i j} \sum_{k=1}^{n}\left(p_{21}\right)_{j k} \alpha_{k}=\left(p_{21}\right)_{j i} \alpha_{i} \quad \text { for } \mathrm{i}=1,2, \ldots, n ; j=1,2, \ldots, m
$$

Summing up Eq.(11) with respect to $\mathfrak{j}$, we obtain

$$
\sum_{k=1}^{n}\left(\sum_{j=1}^{m}\left(p_{12}\right)_{i j}\left(p_{21}\right)_{j k}\right) \alpha_{k}=\alpha_{i} \quad \text { for } i=1,2, \ldots, n
$$

or

$$
P_{12} P_{21} \alpha=\alpha
$$

where $\alpha=\left(\alpha_{1}, \alpha_{2}, \ldots, \alpha_{n}\right)^{\mathrm{T}}$. Similarly, we can obtain 


$$
P_{21} P_{12} \beta=\beta
$$

where $\beta=\left(\beta_{1}, \beta_{2}, \ldots, \beta_{m}\right)^{\top}$. From Eq. (13), it can be seen that

$$
\mathrm{P}_{21} \mathrm{P}_{12} \mathrm{P}_{21} \alpha=\mathrm{P}_{21} \alpha
$$

This means that $P_{21} \alpha$ is also the solution of Eq. (14). From the uniqueness of solution of Eq. (14), we obtain

or

$$
\beta=P_{21} \alpha
$$

$$
\beta_{j}=\sum_{k=1}^{\mathfrak{n}}\left(p_{21}\right)_{j k} \alpha_{k} \quad \text { for } j=1,2, \ldots, m
$$

Similarly, we can obtain

$$
\alpha=\mathrm{P}_{12} \beta
$$

Thus, $\alpha$ and $\beta$ constitute the solution of Eq. (1). Combining Eqs. (11) and (16); we obtain

$$
\left(p_{12}\right)_{i j} \beta_{j}=\left(p_{21}\right)_{j i} \alpha_{i} \quad \text { for } i=1,2, \ldots, n ; j=1,2, \ldots, m
$$

This means $\alpha$ and $\beta$ also satisfy Definition 1 . Therefore, the sufficiency follows.

It is not easy to check the conditions of Lemma 1 . The following lemma will serve this purpose.

Lemma 2: For non-negative matrices $P_{12}$ and $P_{21}$ there exist positive constants $\alpha_{1}, \alpha_{2}, \ldots, \alpha_{n}$ and $\beta_{1}$, $\beta_{2}, \ldots, \beta_{m}$ such that

and

$$
\alpha_{k}\left(p_{12}\right)_{i j}\left(p_{21}\right)_{j k}=\alpha_{i}\left(p_{12}\right)_{k j}\left(p_{21}\right)_{j i} \quad \text { for } i, k=I, 2, \ldots, n ; j=1,2, \ldots, m
$$

$$
\beta_{k}\left(p_{21}\right)_{i j}\left(p_{12}\right)_{j k}=\beta_{i}\left(p_{21}\right)_{k j}\left(p_{12}\right)_{j i} \quad \text { for } i, k=1,2, \ldots m ; j=1,2, \ldots, n
$$

if and only if the ratios

$$
\frac{\left(\mathrm{p}_{12}\right)_{\mathrm{ij}}\left(\mathrm{p}_{21}\right)_{\mathrm{jk}}}{\left(\mathrm{p}_{12}\right)_{\mathrm{kj}}\left(\mathrm{p}_{21}\right)_{\mathrm{ji}}} \quad \text { for }\left(p_{12}\right)_{k j}\left(p_{21}\right)_{j i} \neq 0 ; i, k=1,2, \ldots, n ; j=1,2, \ldots, m
$$

and

$$
\frac{\left(\mathrm{p}_{21}\right)_{\mathrm{ij}}\left(\mathrm{p}_{12}\right)_{\mathrm{jk}}}{\left(\mathrm{p}_{21}\right)_{\mathrm{kj}}\left(\mathrm{p}_{12}\right)_{\mathrm{ji}}} \quad \text { for }\left(p_{21}\right)_{k j}\left(p_{l 2}\right)_{j i} \neq 0 ; i, k=1,2, \ldots, m_{;} ;=1,2, \ldots, n
$$

do not depend on $j$.

Proof: The necessity is obvious. Now we prove the sufficiency. Suppose

$$
\omega_{i k}=\frac{\left(p_{12}\right)_{i j}\left(p_{21}\right)_{j k}}{\left(p_{12}\right)_{k j}\left(p_{21}\right)_{j i}} \quad \text { for }\left(p_{12}\right)_{k j}\left(p_{21}\right)_{j i} \neq 0 ; i, k=1,2, \ldots, n ; j=1,2, \ldots, m
$$

Since the ratios

$$
\frac{\left(p_{12}\right)_{i j}\left(p_{21}\right)_{j k}}{\left(p_{12}\right)_{k j}\left(p_{21}\right)_{j i}} \quad \text { for }\left(p_{12}\right)_{k j}\left(p_{21}\right)_{j i} \neq 0 ; i, k=1,2, \ldots, n ; j=1,2, \ldots, m
$$

do not depend on $j$, we see that

$$
\omega_{i k}=\omega_{i t} \omega_{t k} \quad \text { for } i, k, t=1,2, \ldots, n
$$

Therefor, the matrix $A=\left(\omega_{\mathrm{ik}}\right)$ is consistent and there exist positive constants $\alpha_{1}, \alpha_{2}, \ldots, \alpha_{n}$ such that (Saaty, 1990)

$$
\omega_{\mathrm{ik}}=\frac{\alpha_{\mathrm{i}}}{\alpha_{\mathrm{k}}} \quad \mathrm{i}, \mathrm{k}=1,2, \ldots, \mathrm{n}
$$


From Eqs. (20) and (21) we obtain

or

$$
\frac{\alpha_{i}}{\alpha_{k}}=\frac{\left(p_{12}\right)_{i j}\left(p_{21}\right)_{j k}}{\left(p_{12}\right)_{k j}\left(p_{21}\right)_{j i}} \quad \text { for }\left(p_{12}\right)_{k j}\left(p_{21}\right)_{j i} \neq 0 ; i, k=1,2, \ldots, n ; j=1,2, \ldots, m
$$

$$
\alpha_{\mathrm{k}}\left(\mathrm{p}_{12}\right)_{\mathrm{ij}}\left(\mathrm{p}_{21}\right)_{\mathrm{jk}}=\alpha_{\mathrm{i}}\left(\mathrm{p}_{12}\right)_{\mathrm{kj}}\left(\mathrm{p}_{21}\right)_{\mathrm{ji}} \quad \text { for } \mathrm{i}, \mathrm{k}=1,2, \ldots, \mathrm{n} ; \mathrm{j}=1,2, \ldots, \mathrm{m}
$$

Similarly, we can show that there exist positive constants $\beta_{1}, \beta_{2}, \ldots, \beta_{m}$ such that

$$
\beta_{\mathrm{k}}\left(\mathrm{p}_{21}\right)_{\mathrm{ij}}\left(\mathrm{p}_{\mathrm{i} 2}\right)_{\mathrm{jk}}=\beta_{\mathrm{i}}\left(\mathrm{p}_{2 \mathrm{i}}\right)_{\mathrm{kj}}\left(\mathrm{p}_{\mathrm{i} 2}\right)_{\mathrm{ji}} \quad \text { for } \mathrm{i}, \mathrm{k}=1,2, \ldots \mathrm{m} ; \mathrm{j}=1,2, \ldots, \mathrm{n}
$$

Hence the sufficiency follows.

Combining Lemmas 1 and 2, we have the following theorem.

Theorem 2: When the inter-impact between $L_{1}$ and $L_{2}$ is irreducible with Property $A$, the solution of Eq. (1) is a solution of Definition 1 if and only if, for coefficient matrices $P_{12}$ and $P_{21}$, the ratios

$$
\frac{\left(\mathrm{p}_{12}\right)_{\mathrm{ij}}\left(\mathrm{p}_{21}\right)_{\mathrm{jk}}}{\left(\mathrm{p}_{12}\right)_{\mathrm{kj}}\left(\mathrm{p}_{21}\right)_{\mathrm{ji}}} \quad \text { for }\left(p_{12}\right)_{k j}\left(p_{21}\right)_{j i} \neq 0 ; i, k=1,2, \ldots, n ; j=1,2, \ldots, m
$$

and

$$
\frac{\left(\mathrm{p}_{21}\right)_{\mathrm{ij}}\left(\mathrm{p}_{12}\right)_{\mathrm{jk}}}{\left(\mathrm{p}_{21}\right)_{\mathrm{kj}}\left(\mathrm{p}_{12}\right)_{\mathrm{ji}}} \quad \text { for }\left(p_{2 \nu_{k j}}\left(p_{l 2}\right)_{j i} \neq 0 ; i, k=1,2, \ldots, m ; j=1,2, \ldots, n\right.
$$

do not depend on $j$.

Corollary 2: The inter-impact between $L 1$ and $L 2$ is a Bayes loop.if and only if, for matrices $P_{12}$ and $P_{2 J}$, the ratios

$$
\frac{\left(\mathrm{p}_{12}\right)_{\mathrm{ij}}\left(\mathrm{p}_{21}\right)_{\mathrm{jk}}}{\left(\mathrm{p}_{12}\right)_{\mathrm{kj}}\left(\mathrm{p}_{21}\right)_{\mathrm{ji}}} \quad \text { for }\left(p_{l 2}\right)_{k j}\left(p_{22}\right)_{j i} \neq 0 ; i, k=1,2, \ldots, n ; j=1,2, \ldots, m
$$

and

$$
\frac{\left(\mathrm{p}_{21}\right)_{\mathrm{ij}}\left(\mathrm{p}_{12}\right)_{\mathrm{jk}}}{\left(\mathrm{p}_{21}\right)_{\mathrm{kj}}\left(\mathrm{p}_{\mathrm{i} 2}\right)_{\mathrm{ji}}} \quad \text { for }\left(p_{2 \nu_{k j}}\left(p_{l 2}\right)_{j i} \neq 0 ; i, k=1,2, \ldots, m ; j=1,2, \ldots, n\right.
$$

do not depend on $j$.

Assume that $L_{1}=\left\{\theta_{1}, \theta_{2}, \ldots, \theta_{n}\right\}$ is the set of states of nature and let $L_{2}=\left\{x_{1}, x_{2}, \ldots, x_{m}\right\}$ be the sample space from which observations are drawn at random. Let $P_{21}=\left(\left(p_{21}\right)_{i j}\right)=\left(p\left(x_{j} \mid \theta_{j}\right)\right)$ be the $m \times n$ column stochastic matrix of likelihoods, let $P_{12}=\left(\left(p_{12}\right)_{i j}\right)=\left(p\left(\theta_{i} \mid x_{j}\right)\right)$ be the $n \times m$ column stochastic matrix of posterior probabilities, $P_{1}=\left(\left(p_{1}\right)_{j}\right)=\left(p\left(\theta_{i}\right)\right)$ be the $n \times 1$ vector of prior probabilities, and $P_{2}=\left(\left(p_{2}\right)_{i}\right)=$ $\left(p\left(x_{i}\right)\right)$ be the $m \times 1$ vector of the marginal probabilities of $x_{i}$. We have

$$
p\left(\theta_{i} \mid x_{j}\right) p\left(x_{j}\right)=p\left(x_{j} \mid \theta_{i}\right) p\left(\theta_{i}\right) \quad \text { for } i=1,2, \ldots, n ; j=1,2, \ldots, m
$$

that is, Definition 1 is satisfied. By Theorem 2, we have the following corollary.

Corollary 3: The ratios

do not depend on $j$.

$$
\frac{\mathrm{p}\left(\theta_{\mathrm{i}} \mid \mathrm{x}_{\mathrm{j}}\right) \mathrm{p}\left(\mathrm{x}_{\mathrm{j}} \mid \theta_{\mathrm{k}}\right)}{\mathrm{p}\left(\theta_{\mathrm{k}} \mid \mathrm{x}_{\mathrm{j}}\right) \mathrm{p}\left(\mathrm{x}_{\mathrm{j}} \mid \theta_{\mathrm{i}}\right)} \text { and } \frac{\mathrm{p}\left(\mathrm{x}_{\mathrm{i}} \mid \theta_{\mathrm{j}}\right) \mathrm{p}\left(\theta_{\mathrm{j}} \mid \mathrm{x}_{\mathrm{k}}\right)}{\mathrm{p}\left(\mathrm{x}_{\mathrm{k}} \mid \theta_{\mathrm{j}}\right) \mathrm{p}\left(\theta_{\mathrm{j}} \mid \mathrm{x}_{\mathrm{i}}\right)}
$$


Using Theorem 2, we find a simple method of finding $a$ and $b$ that satisfy Eq. (1) when the conditions of Theorem 2 are satisfied. In fact, in this situation there exist positive constants $\alpha_{1}, \alpha_{2}, \ldots, \alpha_{n}$ and $\beta_{1}$, $\beta_{2}, \ldots, \beta_{\mathrm{m}}$ such that

and

$$
\frac{\alpha_{i}}{\alpha_{k}}=\frac{\left(p_{12}\right)_{i j}\left(p_{21}\right)_{j k}}{\left(p_{12}\right)_{k j}\left(p_{21}\right)_{j i}} \quad \text { for }\left(p_{12}\right)_{k j}\left(p_{21}\right)_{j i} \neq 0 ; i, k=1,2, \ldots, n ; j=1,2, \ldots, m
$$

$$
\frac{\beta_{i}}{\beta_{k}}=\frac{\left(p_{21}\right)_{i j}\left(p_{12}\right)_{j k}}{\left(p_{21}\right)_{k j}\left(p_{12}\right)_{j i}} \text { for }\left(p_{21}\right)_{k j}\left(p_{12}\right)_{j i} \neq 0 ; i, k=1,2, \ldots, m ; j=1,2, \ldots, n
$$

From the ratios of the right side of Eq. (22) and those of the right side of Eq. (23) we can obtain (except a constant factor) $\alpha_{1}, \alpha_{2}, \ldots, \alpha_{n}$ and $\beta_{1}, \beta_{2}, \ldots, \beta_{m}$, respectively. Normalizing them we can obtain $a$ and $b$. Here is an example.

Example 3: Suppose, in Eq. (1),

$$
P_{12}=\left(\begin{array}{llll}
0.4167 & 0.5769 & 0.7143 & 0.2273 \\
0.2500 & 0.1154, & 0.2143 & 0.6818 \\
0.3333 & 0.3077 & 0.0714 & 0.0909
\end{array}\right) \text { and } P_{21}=\left(\begin{array}{ccc}
0.2 & 0.2 & 0.4 \\
0.3 & 0.1 & 0.4 \\
0.4 & 0.2 & 0.1 \\
0.1 & 0.5 & 0.1
\end{array}\right)
$$

It is easy to verify that the conditions of Theorem 2 are satisfied. Then, by normalizing the ratios

$$
\frac{a_{2}}{a_{1}}=\frac{\left(p_{12}\right)_{2 j}\left(p_{21}\right)_{j 1}}{\left(p_{12}\right)_{j j}\left(p_{21}\right)_{j 2}}=0.6, \frac{a_{3}}{a_{1}}=\frac{\left(p_{12}\right)_{3 j}\left(p_{21}\right)_{j 1}}{\left(p_{12}\right)_{j j}\left(p_{21}\right)_{j 3}}=0.4 \text { and } \frac{a_{1}}{a_{1}}=1
$$

we obtain $\mathrm{a}_{1}=0.5, \mathrm{a}_{2}=0.3$ and $\mathrm{a}_{3}=0.2$. Also, by normalizing the ratios

$$
\begin{aligned}
& \frac{b_{2}}{b_{1}}=\frac{\left(p_{21}\right)_{2 j}\left(p_{12}\right)_{j 1}}{\left(p_{21}\right)_{1 j}\left(p_{12}\right)_{j 2}}=1.0833, \frac{b_{3}}{b_{1}}=\frac{\left(p_{21}\right)_{3 j}\left(p_{12}\right)_{j 1}}{\left(p_{21}\right)_{1 j}\left(p_{12}\right)_{j 3}}=1.1667, \\
& \frac{b_{4}}{b_{1}}=\frac{\left(p_{21}\right)_{4 j}\left(p_{12}\right)_{j 1}}{\left(p_{21}\right)_{1 j}\left(p_{12}\right)_{j 4}}=0.9167 \text { and } \frac{b_{1}}{b_{1}}=1
\end{aligned}
$$

we obtain $b_{1}=0.24, b_{2}=0.26, b_{3}=0.28$ and $b_{4}=0.22$. Thus the vectors $a=\left(a_{1}, a_{2}, a_{3}\right)^{T}$ and $b=\left(b_{1}, b_{2}, b_{3}, b_{4}\right)^{\frac{T}{T}}$ form the solution of Eq. (1).

\section{Conclusion}

In this paper, some problems about the solution of a superhierarchy with a Bayes loop are discussed and a simple method of finding the solution is introduced. Theorem 2 shows the relationship between the likelihoods $\left(\left(\mathrm{p}_{21}\right)_{i j}\right)$ and the posterior probabilities $\left(\left(\mathrm{p}_{12}\right)_{i j}\right)$. It is a supplement to Bayes Theorem. The results obtained here can be extended to some more general situations (a superhierarchy with several Bayes loops, for example), which can be the topics for further research.

\section{Reference}

Saaty, T.L., (1994) Fundamentals of Decision Making and Priority Theory with the Analytic Hierarchy Process, Chapter 8, RWS Publication, Pittsburgh, USA 
Gantmacher, F.R., (1977) Matrix Theory, Chapter 13, Chelsea Publishing Company, New York, N.Y., USA

Saaty, T.L., (1990) Multicriteria Decision Making, Chapter 3, RWS Publication, Pittsburgh, USA 